5.

\title{
Addizione alla Memoria intitolata: Nuove applicazioni del Calcolo Integrale relative alla quadratura delle superficie curve e cubatura de solidi, inscritta nel tom. 31 di questo giornale pag 12.
}

(Dal Sign. D. Barnaba Tortolini, prof. di matematiche trasc. a l'Università di Roma.)

1. Se da un punto fisso preso nello spazio si abbassino delle perpendicolari sui piani tangenti di una superficie curva data, il luogo geometrico dei piedi di queste perpendicolari sarà una nuova superficie curva dipendente dalla scelta della prima. Cosi supponendo che il punto fisso coincida con il centro dell'ellissoide, e delle due iperboloidi, il luogo geometrico della projezione ortogonale di questo centro sui respettivi piani tangenti si riduce a tre superficie del quarto ordine, che noi abbiamo già esaminato per ciò, che riguarda specialmente la loro quadratura, ed il volume terminato dalla stessa superficie nella Memoria in data del $1^{\circ}$ Novembre 1844 , ed alla quale appartiene la presente addizione. Tornando alla considerazione di una qualsiasi superficie curva, supponiamo, che si faccia derivare da questa, una serie di superficie curve, le quali si succedano con la stessa legge di sopra espressa, noi chiameremo queste superficie, come il $\mathrm{Sig}^{\mathrm{r}}$ William Roberts di Dublino ha fatto per le curve piane, superficie curve del sistema positivo, od anche superficie curve positive ${ }^{*}$ ).

$2^{\circ}$. Imaginando ora una superficie curva, che sia costantemente toccata da piani perpendicolari, condolti all' estremità dei raggi veltori di una superficie data; supponiamo che dalla nuova superficie curva se ne faccia derivare una terza per un modo simile di generazione, e cosi di seguito: noi otterremo

*) Il Sigr William. Roberts, in una Memoria inscritta nel giornale del Sig ${ }^{\mathrm{r}}$ Liouville per il mese di Maggio 1845, si è occupato della rettificazione delle curve, luoge geometrico dei piedi delle perpendicolari abbassale da un punto fisso sopra le tangenti di una curva data. Fin dal settembre 1844 io avea già pubblicato nel giornale Arcadico una Memoria sopra la rettificazione di due curve del quarto ordine provenienti dalla projezione del centro dell' ellissi, e dell' iperbola sulle tangenti. La lettura della Memoria del Sig $^{r}$ Roberts mi hà suggerito nuove ricerche sopra la rellificazione delle curve, e che trovarsi esposte nel giornale arcadico per l'ultimo trimestre 1845.

Crelle's Journal f. d. M. Bd. XXXIV. Heft 2. 
altrettante superficie curve, che come il medesimo Sig $^{r}$ Roberts ha praticato per le curve piane, chiameremo superficie curve del sistema negativo, o semplicemente superficie curve negative ${ }^{*}$ ). Ciò posto, come nel sistema positivo una qualunque delle superficie d̀ una derivata positiva della sua antecedente, cosi essa stessa sarà una superficie derivata negativa della sua consecutiva: viceversa, come una qualunque delle superficie nel sistema negativo è una derivata negativa della sua antecedente, cosi essa stessa sarà una superficie derivata positiva della sua consecutiva. Le superficie curve del doppio sistema godono di un' interessante proprielà che è ben di conoscere. Se dal consueto punto fisso si conducano i raggi vettori ai punti corrispondenti di ciascuna superficie nel doppio sistema, condotti i piani tangenti ai medesimi punti saranno tutti eguali gli angoli formati dalla stessa parte dai piani tangenti con i respettivi raggi vettori. L'enunciata proprietà porge un' estensione ad una proposizione somigliante trovata dal Sigr Roberts per le curve piane, la quale gli ha somministrato una formola generale per la rettificazione di una qualunque delle curve del doppio sistema. Quantunque sia assai facile di stabilire le formole generali dalle quali dipende la natura delle superficie derivate positive, e negative, contuttociò non sarà inutile che in questa addizione ci fermiamo primieramente sopra la maniera di rappresentare l'equazioni delle medesime, facendo uso delle note equazioni del piano tangente e della normale: la scelta del punto fisso, dal quale si devono abbassare le perpendicolari essendo in nostro arbitrio, noi per non complicare di soverchio le formole, prenderemo questo punto per l'origine delle coordinate. In appresso risolveremo un problema sulla quadratura di qualche superficie negativa derivata dal centro di una superficie del secondo ordine: Problemi simili di quadratura, e cubatura si trovano già risoluti per le superficie positive nella citata Memoria.

$3^{\circ}$. Sieno $x, y, z$ le coordinate ortogonali di un punto qualunque di una superficie curva rappresentata dall' equazione generale

$$
\boldsymbol{u}=\boldsymbol{H}(\boldsymbol{x}, y, \boldsymbol{z})=0 .
$$

Conducendo per il punto $(x, y, z)$ un piano tangente, e chiamando $\boldsymbol{X}, \boldsymbol{Y}, \boldsymbol{Z}$

*) Il Sigr Roberts nella citata Memoria si occupa ancora della rettificazione delle curve, alle quali sieno costantemente tangenti le perpendicolari condotle all' estremità dei raggi veltori, e da esso chiamate curve negative. Dimostra che se $\boldsymbol{u}$ indichi il numero delle curve derivate, la formola per la rettificazione delle curve positive passa ad essere quella delle negative col solo cangiare $n$ in, $-n$. Tal' è il motivo della denominazione di curve positive, e negative. 
le coordinate di un punto qualunque di questo piano, si avrà per la sua equazione

$$
(\boldsymbol{X}-\boldsymbol{x}) \partial_{x} u+(\boldsymbol{Y}-y) \partial_{y} u+(\boldsymbol{Z}-\boldsymbol{z}) \partial_{z} u=0
$$

ove $\partial_{x} u, \partial_{y} u, \partial_{z} u$ sono secondo le consuete notazioni le derivate parziali dell' equazione $u=0$. Se ora dall' orgine delle coordinate si abbassi una perpendicolare sulla direzione del piano tangente, le sue equazioni saranno

$$
\frac{\boldsymbol{X}}{\partial_{x} u}=\frac{\boldsymbol{Y}}{\partial_{y} u}=\frac{\boldsymbol{Z}}{\partial_{z} u} \text {. }
$$

Nella coesistenza dell' equazioni del piano tangente, e della normale per i medesimi valori di $\boldsymbol{X}, \boldsymbol{Y}, \boldsymbol{Z}$ trovasi l'equazione della nuova superficie, luogo geometrico dei piedi delle perpendicolari abbassate dall'orgine delle coordinate sopra tutti i piani tangenti: siccome poi le tre variabili $x, y, z$ potremo sempre supporre ridotle a due di quelle, o a due nuove, cosi l'eliminazione delle due ultime variabili fra le riportale equazioni ci farà giungere ad una equazione unica fra le coordinate $\boldsymbol{X}, \boldsymbol{Y}, \boldsymbol{Z}$ quale apparterrà alla superficie in questione. L'eliminazione delle variabili presentando per lo più grandi difficoltà, proporremo piuttosto un' altro metodo, che ci fara scuoprire egualmente bene l'indole della superficie. Osserviamo primieramente che i valori di $\boldsymbol{X}, \boldsymbol{Y}, \boldsymbol{Z}$ essendo di forma lineare in ambedue l'equazioni, si del piano tangente, che della normale, ricaveremo facilmente

$$
\frac{X}{\partial_{x} u}=\frac{\boldsymbol{Y}}{\partial_{y} u}=\frac{\boldsymbol{Z}}{\partial_{z} u}=\frac{\boldsymbol{X} \partial_{x} u+\boldsymbol{Y} \partial_{y} u+\boldsymbol{Z} \partial_{z} u}{\left(\partial_{x} u\right)^{2}+\left(\partial_{y} u\right)^{2}+\left(\partial_{z} u\right)^{2}}
$$

od anche per l'equazione del piano tangente,

d'onde

$$
\frac{\boldsymbol{X}}{\partial_{x} u}=\frac{\boldsymbol{Y}}{\partial_{y} u}=\frac{\boldsymbol{Z}}{\partial_{z} u}=\frac{x \partial_{x} u+y \partial_{y} u+z \partial_{z} u}{\left(\partial_{x} u\right)^{2}+\left(\partial_{y} u\right)^{2}+\left(\partial_{z} u\right)^{2}}
$$

$$
\begin{gathered}
\boldsymbol{X}=\frac{\partial_{x} u\left(x \partial_{x} u+y \partial_{y} u+z \partial_{z} u\right)}{\left(\partial_{x} u\right)^{2}+\left(\partial_{y} u\right)^{2}+\left(\partial_{z} u\right)^{2}}, \quad \boldsymbol{Y}=\frac{\partial_{y} u\left(x \partial_{x} u+y \partial_{y} u+z \partial_{z} u\right)}{\left(\partial_{x} u\right)^{2}+\left(\partial_{y} u\right)^{2}+\left(\partial_{z} u\right)^{2}} \\
\boldsymbol{Z}=\frac{\partial_{z} u\left(x \partial_{x} u+y \partial_{y} u+z \partial_{z} u\right)}{\left(\partial_{x} u\right)^{2}+\left(\partial_{y} u\right)^{2}+\left(\partial_{z} u\right)^{2}}
\end{gathered}
$$

Tali sono i valori delle coordinate $\boldsymbol{X}, \boldsymbol{Y}, \boldsymbol{Z}$ della superficie derivata positiva in funzione delle coordinate $x, y, z$ del punto corrispondente nella superficie primitiva. Le tre variabili $x, y, z$ potendosi ridurre a sole due, l'equazione della superficie sarà la risultante dall' eliminazione di queste due ultime variabili. Volendo calcolare il raggio $\boldsymbol{R}$ condotto dall' orgine al punto $(\boldsymbol{X}, \boldsymbol{Y}, \boldsymbol{Z})$, si ricaverà

$$
\boldsymbol{R}= \pm \frac{\left(x \partial_{x} u+y \partial_{y} u+z \partial_{z} u\right)}{\sqrt{\left(\left(\partial_{x} u\right)^{2}+\left(\partial_{y} u\right)^{2}+\left(\partial_{y} u\right)^{2}\right)}}
$$


La distanza $\boldsymbol{R}$ non è altro che la perpendicolare abbassata dall' origine sopra la direzione del piano tangente la superficie curva nel punto $(x, y, z)$. Quando l'equazione della superficie fosse risoluta rapporto ad una delle variabili $z$ in modo da essere

$$
u=f(x, y, z)-z=0,
$$

allora, ponendo per le derivate parziali:

$$
\partial_{x} u=z^{\prime}, \quad \partial_{y} u=z_{1}, \quad \partial_{z} u=-1
$$

si troverà

$$
X=\frac{z^{\prime}\left(x z^{\prime}+y z_{1}-z_{1}\right)}{1+z^{\prime 2}+z_{1}^{2}}, \quad Y=\frac{z_{1}\left(x z^{\prime}+y z_{1}-z\right)}{1+z^{\prime 2}+z_{1}^{2}}, \quad Z=\frac{-\left(x z^{\prime}+y z_{1}-z\right)}{1+z^{\prime 2}+z_{1}^{2}} .
$$

Il raggio $\boldsymbol{R}$ diviene

$$
\boldsymbol{R}= \pm \frac{\left(x z^{\prime}+y z_{1}-z\right)}{\sqrt{\left(1+z^{\prime 2}+z_{1}^{2}\right)}}
$$

Le precedenti formole sono tutte quelle che possono occorrere volendo far uso delle coordinate ortogonali.

$4^{\circ}$. Per mostrare una qualche applicazione, prendiamo un' ellissoide

$$
\frac{x^{2}}{a^{2}}+\frac{y^{2}}{b^{2}}+\frac{z^{2}}{c^{2}}=1
$$

avremo dalla derivazione dell' equazione $u=0$,

$$
\partial_{x} u=\frac{2 x}{a^{2}}, \quad \partial_{y} u=\frac{2 y}{b^{2}}, \quad \partial_{z} u=\frac{2 z}{c^{2}},
$$

d'onde

$$
X=\frac{x}{a^{2}\left(\frac{x^{2}}{a^{4}}+\frac{y^{2}}{b^{4}}+\frac{z^{2}}{c^{4}}\right)}, \quad Y=\frac{y}{b^{2}\left(\frac{x^{2}}{a^{4}}+\frac{y^{2}}{b^{4}}+\frac{z^{2}}{c^{4}}\right)}, \quad Z=\frac{z}{c^{2}\left(\frac{x^{2}}{a^{4}}+\frac{y^{2}}{b^{4}}+\frac{z^{2}}{c^{4}}\right)}
$$

ed insieme

$$
\boldsymbol{R}=\frac{1}{\sqrt{\left(\frac{x^{2}}{a^{4}}+\frac{y^{2}}{b^{4}}+\frac{z^{2}}{c^{4}}\right)}}
$$

Ognun vede che il raggio $\boldsymbol{R} \dot{e}$ la nota espressione della perpendicolare abhassata dal centro dell'ellissoide sopra il piano tangente. L'eliminazione delle $x, y, z$ si rende qui assai facile: infatti moltiplicando respettivamente i vaTori di $\boldsymbol{X}, \boldsymbol{Y}, \boldsymbol{Z}$ per $a, b, c$, ed elevando al quadrato, si troverà dalla somma dei quadrati

$$
a^{2} X^{2}+b^{2} Y^{2}+c^{2} Z^{2}=\frac{1}{\left(\frac{x^{2}}{a^{4}}+\frac{y^{2}}{b^{4}}+\frac{z^{2}}{c^{4}}\right)^{2}}
$$


b. Tortolini, quad. delle superficie curve et cubat. de solidi.

Ora il secondo membro $\dot{e}$ evidentemente la quarta potenza del raggio

$$
\boldsymbol{R}=\sqrt{ }\left(\boldsymbol{X}^{2}+\boldsymbol{Y}^{2}+\boldsymbol{Z}^{2}\right)
$$

e perciò

$$
\left(\boldsymbol{X}^{2}+\boldsymbol{Y}^{2}+\boldsymbol{Z}^{2}\right)^{2}=a^{2} \boldsymbol{X}^{2}+\boldsymbol{b}^{2} \boldsymbol{Y}^{2}+\boldsymbol{c}^{2} \boldsymbol{Z}^{2} .
$$

Quest' equazione di quarto grado appartiene alla prima superficie derivata positiva dal centro dell' ellissoide ed è precisamente quella alla quale giunsi nella mia precedente Memoria per considerazioni alquanto diverse. Avuto riguardo alla doppia espressione di $\boldsymbol{R}$, dai valori di $\boldsymbol{X}, \boldsymbol{Y}, \boldsymbol{Z}$ si dedurrà reciprocamente

$$
x=\frac{a^{2} X}{X^{2}+Y^{2}+Z^{2}}, \quad y=\frac{b^{2} Y}{X^{2}+Y^{2}+Z^{2}}, \quad z=\frac{c^{2} Z}{X^{2}+Y^{2}+Z^{2}} .
$$

Sostituendo poi questi valori o nell' equazione dell' ellissoide, o nell' espressione della distanza $\boldsymbol{R}$, si giungerà in ambedue modi alla riportata equazione della superficie del quarto ordine. Osservando in fine che l'equazione dell' ellissoide vien verificata dai valori

$$
x=a \cos \theta, \quad y=b \operatorname{sen} \theta \cos \omega, \quad z=c \operatorname{sen} \theta \operatorname{sen} \omega,
$$

otterremo per $\boldsymbol{X}, \boldsymbol{Y}, \boldsymbol{Z}$ le formole

$$
\begin{aligned}
& X=\frac{a b^{2} c^{2} \cos \theta}{b^{2} c^{2} \cos ^{2} \theta+a^{2} c^{2} \operatorname{sen}^{2} \theta \cos ^{2} \omega+a^{2} b^{2} \operatorname{sen}^{2} \theta \operatorname{sen}^{2} \omega}, \\
& \boldsymbol{Y}=\frac{b a^{2} c^{3} \operatorname{sen} \theta \cos \omega}{b^{2} c^{2} \cos ^{2} \theta+a^{2} c^{2} \operatorname{sen}^{2} \theta \cos ^{2} \omega+a^{2} b^{2} \operatorname{sen}^{2} \theta \operatorname{sen}^{2} \omega}, \\
& \mathbf{C}=\frac{c a^{2} b^{2} \operatorname{sen} \theta \operatorname{sen} \omega}{b^{2} c^{2} \cos ^{2} \theta+a^{2} c^{2} \operatorname{sen}^{2} \theta \cos ^{2} \omega+a^{2} b^{2} \operatorname{sen}^{2} \theta \operatorname{sen}^{2} \omega} .
\end{aligned}
$$

Queste nouve espressioni potrebbero utilmente adoprarsi nella ricerca della quadratura della superficie, o nella cubatura del solido.

$5^{\circ}$. Volendo proseguire alla ricerca della seconda superficie derivata positiva dal centro dell' ellissoide, mutereme $X, Y, Z$ in $x, y, z$ cosicche prendendo l'equazione alla superficie del quarto ordine

$$
\left(x^{2}+y^{2}+z^{2}\right)^{2}=a^{2} x^{2}+b^{2} y^{2}+c^{2} z^{2},
$$

si abbia dalle derivate parziali:

$$
\begin{gathered}
\partial_{x} u=2 x\left(x^{2}+y^{2}+z^{2}-a^{2}\right), \quad \partial_{y} u=2 y\left(x^{2}+y^{2}+z^{2}-b^{2}\right), \\
\partial_{z} u=2 z\left(x^{2}+y^{2}+z^{2}-c^{2}\right)
\end{gathered}
$$

quindi ritenuto $r=\sqrt{ }\left(x^{2}+y^{2}+z^{2}\right)$, si ricaverà dalle formole generali dell' antecedente parag. $3^{\circ}$ :

$X=\frac{r^{4} x\left(2 r^{2}-a^{2}\right)}{a^{4} x^{2}+b^{4} y^{2}+c^{4} z^{2}}, \quad Y=\frac{r^{4} y\left(2 r^{2}-b^{2}\right)}{a^{4} x^{2}+b^{4} y^{2}+c^{4} z^{2}}, \quad Z=\frac{r^{4} z\left(2 r^{2}-c^{2}\right)}{a^{4} x^{2}+b^{4} y^{2}+c^{4} z^{2}}$. 
Il corrispondente raggio $\boldsymbol{R}$ sarà egualmente

$$
\boldsymbol{R}=\frac{r^{4}}{\sqrt{\left(a^{4} x^{2}+b^{4} y^{2}+c^{4} z^{2}\right)}} .
$$

Qui l'eliminazione delle $x, y, z$ si rende difficile, e l'equazione della nuova superficie fra le coordinate $\boldsymbol{X}, \boldsymbol{Y}, \boldsymbol{Z}$ sarà di un grado molto elevato: quante volte adunque non venga data l'equazione della superficie fra le coordinate ortogonali, sarà alquanto complicato il proseguire alla ricerca dei valori delle coordinate della nuova superficie derivata, e converrebbe adoprare delle trasformazioni, le quali hanno luogo nel cangiamente delle variabili indipendenti. Chi volesse esprimere i valori di $\boldsymbol{X}, \boldsymbol{Y}, \boldsymbol{Z}$ e della distanze $\boldsymbol{R}$ per mezzo delle coordinate, e del raggio corrispondenti al punto $\left(x^{\prime}, y^{\prime}, \boldsymbol{z}^{\prime}\right)$ dell' ellissoide, avrà a sostituire nelle precedenti formole i respettivi valori di $x, y, z, r$ di già oltenuti al principio del parag. $4^{\circ}$, vale a dire

$$
\begin{array}{ll}
x=\frac{x^{\prime}}{a^{2}\left(\frac{x^{\prime 2}}{a^{4}}+\frac{y^{\prime 2}}{b^{4}}+\frac{z^{\prime 2}}{c^{4}}\right)}, & y=\frac{y^{\prime}}{b^{2}\left(\frac{x^{\prime 2}}{a^{4}}+\frac{y^{\prime 2}}{b^{4}}+\frac{z^{\prime 2}}{c^{4}}\right)}, \\
z=\frac{z^{\prime}}{c^{2}\left(\frac{x^{\prime 2}}{a^{4}}+\frac{y^{\prime 2}}{b^{4}}+\frac{z^{\prime 2}}{c^{4}}\right)}, & r=\frac{1}{\sqrt{\left(\frac{x^{\prime 2}}{a^{4}}+\frac{y^{\prime 2}}{b^{4}}+\frac{z^{\prime 2}}{c^{4}}\right)}} ;
\end{array}
$$

mà sarà qui opportuno di mettere a profitto la proprietà menzionata al parag. $2^{\circ}$, della quale godono le superficie derivate, e relativa all' eguaglianza degli angoli formati dai piani tangenti con $\mathrm{i}$ raggi vettori. Sia infatti $\rho$ il raggio vettore condotto dal centro dell' ellissoide al punto $\left(x^{\prime}, y^{\prime}, z^{\prime}\right)$, e sieno $r, \boldsymbol{R}$ i raggi delle due superficie derivate condotti ai punti $(x, y, \boldsymbol{z}),(\boldsymbol{X}, \boldsymbol{Y}, \boldsymbol{Z})$, e sia infine $\alpha$ uno degli angoli formati dal piano tangente con il raggio vettore, si avrà

quindi

$$
\boldsymbol{r}=\rho \operatorname{sen} \alpha, \quad \boldsymbol{R}=r \operatorname{sen} \alpha,
$$

Pongasi ora per brevità

$$
\boldsymbol{R}=\frac{\boldsymbol{r}^{2}}{\varrho}
$$

$$
\xi=\cos \theta, \quad \eta=\operatorname{sen} \theta \cos \omega, \quad \zeta=\operatorname{sen} \theta \operatorname{sen} \omega,
$$

l'equazione dell' ellissoide porgerà

dalle quali

$$
x^{\prime}=a \xi, \quad y^{\prime}=b \eta, \quad z^{\prime}=c \xi
$$

$$
r=\frac{1}{\sqrt{\left(\frac{\xi^{2}}{a^{2}}+\frac{\eta^{2}}{b^{2}}+\frac{\zeta^{2}}{c^{2}}\right)}}, \quad \rho=\sqrt{ }\left(a^{2} \xi^{2}+b^{2} \eta^{2}+c^{2} \zeta^{2}\right)
$$


e perciò

$$
\boldsymbol{R}=\frac{1}{\left(\frac{\xi^{2}}{a^{2}}+\frac{\eta^{2}}{b^{2}}+\frac{\zeta^{2}}{c^{2}}\right) \sqrt{ }\left(a^{2} \xi^{2}+b^{2} \eta^{2}+c^{2} \zeta^{2}\right)} .
$$

Tal' è il valore di $\boldsymbol{R}$, che si sarebbe potuto ottenere da una successiva sostituzione. I valori poi di $x, y, z$ danno

$$
\begin{gathered}
a^{2} x=r^{2} x^{\prime}, \quad b^{2} y=r^{2} y^{\prime}, \quad c^{2} z=r^{2} z^{\prime}, \\
a^{4} x^{2}+b^{4} y^{2}+c^{4} z^{2}=r^{4} \varphi^{2} .
\end{gathered}
$$

Con queste differenti sostituzioni olteniamo

$$
X=\frac{r^{2} \xi}{a} \cdot \frac{2 r^{2}-a^{2}}{\varrho^{2}}, \quad \boldsymbol{Y}=\frac{r^{2} \eta}{b} \cdot \frac{2 r^{2}-b^{2}}{\varrho^{2}}, \quad Z=\frac{r^{2} \zeta}{c} \cdot \frac{2 r^{2}-c^{2}}{\varrho^{2}} .
$$

Ognun vede che i nuovi valori di $\boldsymbol{X}, \boldsymbol{Y}, \boldsymbol{Z}$ dipendono dopo la sostituzione del valore di $\boldsymbol{r}$ dai soli elementi dell' ellissoide. Infine faremo un' osservazione tutta propria delle applicazioni, che abbiamo scelta. L'equazione alla superficie del quarto ordine

$$
\left(x^{2}+y^{2}+z^{2}\right)^{2}=a^{2} x^{2}+b^{2} y^{2}+c^{2} z^{2}
$$

per la sostituzione di $r^{2}=x^{2}+y^{2}+z^{2}$ si potrà presentare sotto la forma

$$
\frac{x^{2}}{\left(\frac{r^{2}}{a}\right)^{2}}+\frac{y^{2}}{\left(\frac{r^{2}}{b}\right)^{2}}+\frac{z^{2}}{\left(\frac{r^{2}}{c}\right)^{2}}=1,
$$

la quale si verificherà dall' equazioni sferiche

$$
\frac{a x}{r^{2}}=\cos \theta, \quad \frac{b y}{r^{2}}=\operatorname{sen} \theta \cos \omega, \quad \frac{c z}{r^{2}}=\operatorname{sen} \theta \operatorname{sen} \omega,
$$

e che risolute rapporto ad $x, y, z$ coincideranno con i valori di già stabiliti in questo parag. $6^{\circ}$. Le formole generali ottenute al parag. $3^{\circ}$ potranno forse più facilmente prestarsi per la risoluzione di un qualche problema, qualora si faccia uso delle equazioni fra le coordinate polari, ciò che noi verremo brevemente ad indicare. Ritenuto che $\boldsymbol{u}=\mathbf{0}$ sia l'equazione alla superficie primitiva fra le coordinate ortogonali $x, y, z$, supponiamo che sieno funzioni di tre nuove variabili $r, p, q$ si avrà dalle note formole sul cangiamento delle variabili nei differenziali delle funzioni

$$
\begin{aligned}
& \partial_{x} u=\partial_{r} u \partial_{x} r+\partial_{p} u \partial_{x} p+\partial_{q} u \partial_{x} q, \\
& \partial_{y} u=\partial_{r} u \partial_{y} r+\partial_{p} u \partial_{y} p+\partial_{q} u \partial_{y} \boldsymbol{q}, \\
& \partial_{z} u=\partial_{r} u \partial_{z} r+\partial_{p} u \partial_{z} p+\partial_{q} u \partial_{z} \boldsymbol{q} .
\end{aligned}
$$

Riducendosi le variabili $r, p, q$ a tre coordinate polari determinate dall' equazioni

$$
x=r \cos p, \quad y=r \operatorname{sen} p \cos q, \quad z=r \operatorname{sen} p \operatorname{sen} q,
$$


si ricaverà reciprocamente

$$
r^{2}=x^{2}+y^{2}+z^{2}, \quad \cos p=\frac{x}{\sqrt{\left(x^{2}+y^{2}+z^{2}\right)}}, \quad \operatorname{tang} y=\frac{z}{y},
$$

nelle quali eseguendo le derivazioni parziali rapporto ad $x, y, z$ si troverà dalla sostituzione

$$
\begin{aligned}
& \partial_{x} u=\cos p \partial_{r} u-\frac{\operatorname{sen} p}{r} \partial_{p} u, \\
& \partial_{y} u=\operatorname{sen} p \cos \varphi \partial_{r} u+\frac{\cos p \cos q}{r} \partial_{p} u-\frac{\operatorname{sen} q}{r \operatorname{sen} p} \partial_{q} u, \\
& \partial_{z} u=\operatorname{sen} p \operatorname{sen} q \partial_{r} u+\frac{\cos p \operatorname{sen} q}{r} \partial_{p} u+\frac{\cos q}{r \operatorname{sen} p} \partial_{q} u .
\end{aligned}
$$

Queste equazioni di forma lineare rapporlo a tutte le derivate parziali, porgeranno dalla loro risoluzione $\mathrm{i}$ valori delle derivate $\partial_{r} u, \partial_{p} u, \partial_{q} u$ in funzione delle $\partial_{x} u, \partial_{y} u, \partial_{z} u:$ i riportati valori delle derivate danno egualmente

$$
\begin{aligned}
x \partial_{x} u+y \partial_{y} u+z \partial_{z} u & =r \partial_{r} u, \\
\left(\partial_{x} u\right)^{2}+\left(\partial_{y} u\right)^{2}+\left(\partial_{z} u\right)^{2} & =\left(\partial_{r} u\right)^{2}+\frac{\left(\partial_{p} u\right)^{2}}{r^{2}}+\frac{\left(\partial_{q} u\right)^{2}}{r^{2}}
\end{aligned}
$$

Di qui le citate formole del parag. $3^{\circ}$, le quali rappresentano $i$ valori delle coordinate $\boldsymbol{X}, \boldsymbol{Y}, \boldsymbol{Z}$ della prima superficie derivata positiva si trasformeranno in

$$
\begin{aligned}
& \boldsymbol{X}=\frac{r^{2} \partial_{r} u\left(r \cos p \partial_{r} u-\operatorname{sen} p \partial_{p} u\right)}{r^{2}\left(\partial_{r} u\right)^{2}+\left(\partial_{p} u\right)^{2}+\left(\partial_{q} u\right)^{2}}, \\
& \boldsymbol{Y}=\frac{r^{2} \partial_{r} u\left(r \operatorname{sen}^{2} p \cos \eta \partial_{r} u+\operatorname{sen} p \cos p \cos q \partial_{p} u-\operatorname{sen} \eta \partial_{q} u\right)}{r^{2}\left(\partial_{r} u\right)^{2}+\left(\partial_{p} u\right)^{2}+\left(\partial_{q} u\right)^{2}}, \\
& \mathbf{Z}=\frac{r^{2} \partial_{r} u\left(r \operatorname{sen}^{2} p \operatorname{sen} \eta \partial_{r} u+\operatorname{sen} p \cos p \operatorname{sen} q \partial_{p} u+\cos q \partial_{q} u\right)}{r^{2}\left(\partial_{r} u\right)^{2}+\left(\partial_{p} u\right)^{2}+\left(\partial_{q} u\right)^{2}} .
\end{aligned}
$$

Il raggio $\boldsymbol{R}$ diverrà

$$
\boldsymbol{R}= \pm \frac{r^{2} \partial_{r} u}{\sqrt{\left\{r^{2}\left(\partial_{r} u\right)^{2}+\left(\partial_{p} u\right)^{2}+\left(\partial_{q} u\right)^{2}\right\}}}
$$

Per aggiungere ad $\boldsymbol{R}$ le altre coordinate polari, $\boldsymbol{P}, \boldsymbol{Q}$, non avremo che a porre

$$
\boldsymbol{X}=\boldsymbol{R} \cos \boldsymbol{P}, \quad \boldsymbol{Y}=\boldsymbol{R} \operatorname{sen} \boldsymbol{P} \cos \boldsymbol{Q}, \quad \boldsymbol{Z}=\boldsymbol{R} \operatorname{sen} \boldsymbol{P} \operatorname{sen} \boldsymbol{Q},
$$

d' onde si troverebbero due funzioni trigonometriche di $\boldsymbol{P}, \boldsymbol{Q}$ espresse per $r, p, q$. Se l'equazione della superficie primitiva trovasi risoluta rapporto ad una delle variabili $r$, in modo da essere

$$
u=f(p, q)-r=0,
$$

porremo per le derivate parziali

$$
\partial_{r} u=-1, \quad \partial_{p} u=r^{\prime}, \quad \partial_{q} u=r_{1},
$$


ed allora i precedenti valori di $\boldsymbol{X}, \boldsymbol{Y}, \boldsymbol{Z}, \boldsymbol{R}$ si cangieranno in

$$
\begin{aligned}
& \boldsymbol{X}=\frac{r^{2}\left(r \cos p+r^{\prime} \operatorname{sen} p\right)}{r^{2}+r^{\prime 2}+r_{1}^{2}}, \\
& \boldsymbol{Y}=\frac{r^{2}\left(r \operatorname{sen}^{2} p \cos q-r^{\prime} \operatorname{sen} p \cos p \cos q+r_{1} \operatorname{sen} q\right)}{r^{2}+r^{\prime 2}+r_{1}^{2}} \\
& \boldsymbol{Z}=\frac{r^{2}\left(r \operatorname{sen}^{2} p \operatorname{sen} q-r^{\prime} \operatorname{sen} p \cos p \operatorname{sen} q-r_{1} \cos q\right)}{r^{2}+r^{\prime 2}+r_{1}^{2}} \\
& \boldsymbol{R}= \pm \frac{r^{2}}{\sqrt{\left(r^{2}+r^{\prime 2}+r_{1}^{2}\right)}} .
\end{aligned}
$$

La scelta, e l'uso di queste differenti formole dipenderà generalmente dalla natura della questione che s'intraprende a risolvere.

$7^{\circ}$. Passiamo ora alle superficie derivate del sistema negativo. Le superficie curve in questione sono toccate da piani perpendicolari condotti all' estremità dei raggi vettori di una data superficie curva. A questo oggetto sia secondo il consueto $u=0$ l'equazione della superficie primitiva fra le coordinate ortogonali $x, y, \approx$, e riteniamo che il punto fisso dal quale partano i raggi vettori coincida con l'origine. Ciò posto, se all' estremità di un raggio vettore condotto dall' origine al punto $(x, y, z)$ si conduca per lo stesso punto un piano perpendicolare, avremo per la sua equazione.

$$
\text { 1. } \boldsymbol{X} x+\boldsymbol{Y} y+Z_{z}=x^{2}+y^{2}+z^{2},
$$

ove $\boldsymbol{X}, \boldsymbol{Y}, \boldsymbol{Z}$ sono le coordinate di un punto qualunque del piano. Onde queste equazioni possano servire alla risoluzione del proposto problema, converrà differenziarle parzialmente rapporto ad $x$, ed $y$, e quindi dedurre due nuove equazioni risultanti dall' eliminazione dei coefficienti differenziali. Ponendo adunque per le derivate parziali

$$
\frac{\partial z}{\partial x}=\boldsymbol{p}, \quad \frac{\partial z}{\partial y}=\boldsymbol{q},
$$

avremo dall' equazione $u=0$ :

$$
\partial_{x} u+p \partial_{z} u=0, \quad \partial_{y} u+q \partial_{z} u=0,
$$

come dalla (1.) si ricaverà egualmente

$$
X-2 x+(Z-2 z) p=0, \quad Y-2 y+(Z-2 z) \varphi=0 .
$$

Eliminando fra queste ultime quattro le derivate parziali $p$, $q$, si avrà

2. $(X-2 x) \partial_{z} u-(\boldsymbol{Z}-2 z) \partial_{x} u=0, \quad(Y-2 y) \partial_{z} u-(\boldsymbol{Z}-2 z) \partial_{y} u=0$.

L'equazione della nuova superficie sarà la risultante dall' eliminazione delle $x, y, z$ fra la $u=0$ e le (1.), (2.); contullocio come già si ̀̀ praticato Crelle's Journal f. d. M. XXXIV. Heft 2. 
per le superficie del sistema positivo, potremo dedurre i valori delle $\boldsymbol{X}, \boldsymbol{Y}, \boldsymbol{Z}$ in funzione delle $x, y, z$; infatti dalle (2.) abbiamo

$$
\frac{X-2 x}{\partial_{x} u}=\frac{Y-2 y}{\partial_{y} u}=\frac{Z-2 z}{\partial_{z} u}=\frac{X x+Y y+Z z-2\left(x^{2}+y^{2}+z^{2}\right)}{x \partial_{x} u+y \partial_{y} u+z \partial_{z} u},
$$

od anche per la (1.)

$$
\frac{X-2 x}{\partial_{x} u}=\frac{Y-2 y}{\partial_{y} u}=\frac{Z-2 z}{\partial_{z} u}=-\frac{\left(x^{2}+y^{2}+z^{2}\right)}{x \partial_{x} u+y \partial_{y} u+z \partial_{z} u},
$$

dalle quali olteniamo

$$
\begin{aligned}
& \boldsymbol{X}=\frac{\left(x^{2}-y^{2}-z^{2}\right) \partial_{x} u+2 x y \partial_{y} u+2 x z \partial_{z} u}{x \partial_{x} u+y \partial_{y} u+z \partial_{z} u}, \\
& \boldsymbol{Y}=\frac{\left(y^{2}-x^{2}-z^{2}\right) \partial_{y} u+2 x y \partial_{x} u+2 y z \partial_{z} u}{x \partial_{x} u+y \partial_{y} u+z \partial_{z} u}, \\
& \boldsymbol{Z}=\frac{\left(z^{2}-x^{2}-y^{2}\right) \partial_{z} u+2 y z \partial_{y} u+2 x z \partial_{x} u}{x \partial_{x} u+y \partial_{y} u+z \partial_{z} u} .
\end{aligned}
$$

Quando le variabili $\boldsymbol{X}, \boldsymbol{Y}, \boldsymbol{Z}$ si riducano a funzioni date di due sole delle tre $x, y, \approx 0$ almeno dipendano da sole due altre variabili, allora l'eliminazione di queste fra le tre precedenti equazioni si farà giungere all' equazione della prima superficie derivata negativa fra le coordinate $\boldsymbol{X}, \boldsymbol{Y}, \boldsymbol{Z}$. La distanza $R$ dall' origine allo stesso punto $(\boldsymbol{X}, \boldsymbol{Y}, \boldsymbol{Z})$ sarà

$$
\boldsymbol{R}= \pm \frac{\left(x^{2}+y^{2}+z^{2}\right) \sqrt{ }\left(\left(\partial_{x} u\right)^{2}+\left(\partial_{y} u\right)^{2}+\left(\partial_{z} u\right)^{2}\right)}{x \partial_{x} u+y \partial_{y} u+z \partial_{z} u}
$$

Se come già si è fatto al parag. $3^{\circ}$ l'equazione della superficie primitiva fosse risoluta rapporto a $z$, i valori di $\boldsymbol{X}, \boldsymbol{Y}, \boldsymbol{Z}$ prenderanno la forma

$$
\begin{aligned}
& X=\frac{\left(x^{2}-y^{2}-z^{2}\right) z^{\prime}+2 x y z_{1}-2 x z}{x z^{\prime}+y z_{1}-z}, \\
& \boldsymbol{Y}=\frac{\left(y^{2}-x^{2}-z^{2}\right) z_{1}+2 x y z^{\prime}-2 y z}{x z^{\prime}+y z_{1}-z}, \\
& \boldsymbol{Z}=-\frac{\left(z^{2}-x^{2}-y^{2}\right)+2 y z z_{1}+2 x z z^{\prime}}{x z^{\prime}+y z_{1}-z} .
\end{aligned}
$$

Similmente il raggio $\boldsymbol{R}$ si ridurrà ad

$$
\boldsymbol{R}= \pm \frac{\left(x^{2}+y^{2}+z^{2}\right) \sqrt{ }\left(1+z^{\prime 2}+z_{1}^{2}\right)}{x z^{\prime}+y z_{1}-z}
$$

Secondo un'osservazione stabilita al parag. $2^{\circ}$ questa prima superficie derivata negativa, avrà per sua prima derivata positiva la stessa $u=0$, e percio supposto $\boldsymbol{Z}$ funzione di $\boldsymbol{X}, \boldsymbol{Y}$ ed indicando per $\boldsymbol{Z}^{\prime}, \boldsymbol{Z}_{1}$ le derivate parziali rapporto alle stesse $\boldsymbol{X}, \boldsymbol{Y}$; dai precedenti valori di $\boldsymbol{X}, \boldsymbol{Y}, \boldsymbol{Z}$ si devrà avere recipro- 
camente per le ultime formole del parag. $3^{\circ}$ :

$$
\begin{array}{ll}
x=\frac{Z^{\prime}\left(X Z^{\prime}+Y Z_{1}-Z\right)}{1+Z^{\prime 2}+Z_{1}^{2}}, & y=\frac{Z_{1}\left(X Z^{\prime}+Y Z_{1}-Z\right)}{1+Z^{\prime 2}+Z_{1}^{2}}, \\
\approx=-\frac{\left(X Z^{\prime}+Y Z_{1}-Z\right)}{1+Z^{\prime 2}+Z_{1}^{2}}, & r= \pm \frac{\left(X Z^{\prime}+Y Z_{1}-Z\right)}{\sqrt{ }\left(1+Z^{\prime 2}+Z_{1}^{2}\right)}
\end{array}
$$

d'onde ne segue, che questi valori sostituiti nell' espressioni di $\boldsymbol{X}, \boldsymbol{Y}, \boldsymbol{Z}, \boldsymbol{R}$ le renderanno necessariamente identiche.

$8^{\circ}$. Si prenda un' ellissoide per superficie primitiva, e cerchiamo l'equazioni della sua prima derivata negativa dal centro; avremo, come già si è veduto:

$$
\partial_{x} u=\frac{2 x}{a^{2}}, \quad \partial_{y} u=\frac{2 y}{b^{2}}, \quad \partial_{z} u=\frac{2 z}{c^{2}},
$$

d'onde i primi valori di $\boldsymbol{X}, \boldsymbol{Y}, \boldsymbol{Z}$ dell' antecedente parag. diverranno

$$
\begin{aligned}
& X=\frac{x\left(b^{2} c^{2} x^{2}+c^{2}\left(2 a^{2}-b^{2}\right) y^{2}+b^{2}\left(2 a^{2}-c^{2}\right) z^{2}\right)}{a^{2} b^{2} c^{2}}, \\
& \boldsymbol{Y}=\frac{y\left(a^{2} c^{2} y^{2}+c^{2}\left(2 b^{2}-a^{2}\right) x^{2}+a^{2}\left(2 b^{2}-a^{2}\right) z^{2}\right)}{a^{2} b^{2} c^{2}}, \\
& \boldsymbol{Z}=\frac{z\left(a^{2} b^{2} z^{2}+b^{2}\left(2 c^{2}-a^{2}\right) x^{2}+a^{2}\left(2 c^{2}-b^{2}\right) y^{2}\right)}{a^{2} b^{2} c^{2}} .
\end{aligned}
$$

Le variabili $x, y, z$ nell' equazione dell' ellissoide si possono far dipendere da sole due, poiche fatto per brevità

$$
u=\cos p, \quad v=\operatorname{sen} p \cos q, \quad w=\operatorname{sen} p \operatorname{sen} q,
$$

avremo anche

e perciò

$$
x=a u, \quad y=b v, \quad z=c w
$$

$$
\begin{aligned}
& \boldsymbol{X}=\frac{u}{a}\left(a^{2} u^{2}+\left(2 a^{2}-b^{2}\right) v^{2}+\left(2 a^{2}-c^{2}\right) w^{2}\right) \\
& \boldsymbol{Y}=\frac{v}{b}\left(b^{2} v^{2}+\left(2 b^{2}-a^{2}\right) u^{2}+\left(2 b^{2}-c^{2}\right) w^{2}\right), \\
& \boldsymbol{Z}=\frac{w}{c}\left(c^{2} w^{2}+\left(2 c^{2}-a^{2}\right) u^{2}+\left(2 c^{2}-b^{2}\right) v^{2}\right)
\end{aligned}
$$

Se fra queste tre elimineremo le due variabili $p, q$, la nuova equazione fra $\boldsymbol{X}, \boldsymbol{Y}, \boldsymbol{Z}$ apparterrà alla prima superficie derivata negativa dal centro dell' ellissoide. In egual modo si ricaverà dalla formola generale per il räggio vettore $\boldsymbol{R}$ condotto dal centro dell' ellissoide al punto $(X, Y, Z)$ della nuova superficie:

$$
\boldsymbol{R}=\left(x^{2}+y^{2}+z^{2}\right) /\left(\frac{x^{2}}{a^{4}}+\frac{y^{2}}{b^{4}}+\frac{z^{2}}{c^{4}}\right) \text {. }
$$


Indicando con $\boldsymbol{P}$ la perpendicolare abbassata dal centro dell' ellissoide sulla direzione del piano tangente, e ritentito per $\boldsymbol{r}$ il raggio vettore, si avrà facilmente

$$
\boldsymbol{R}=\frac{\boldsymbol{r}^{2}}{\boldsymbol{P}}
$$

e che si ridurrà ad

$$
\boldsymbol{R}=\frac{\left(a^{2} u^{2}+b^{2} v^{2}+c^{2} w^{2}\right) \sqrt[V]{ }\left(b^{2} c^{2} u^{2}+a^{2} c^{2} v^{2}+a^{2} b^{2} w^{2}\right)}{a b c} .
$$

Le precedenti formole troveranno delle utili applicazioni nella quadratura della superficie.

$9^{\circ}$. Riprendiamo per un' istante i valori generali di $\boldsymbol{X}, \boldsymbol{Y}, \boldsymbol{Z}, \boldsymbol{R}$ del parag. $7^{\circ}$, e supponiamo che $x, y, \approx$ si trasformino in coordinate polari $r, p, q$, noi otterremo con facilità

$$
\begin{aligned}
& \boldsymbol{X}=\frac{r \cos p \partial_{r} u+\operatorname{sen} p \partial_{p} u}{\partial_{r} u}, \\
& Y=\frac{r \operatorname{sen} p \cos q \partial_{r} u-\cos p \cos q \partial_{p} u+\frac{\operatorname{sen} q}{\operatorname{sen} p} \partial_{q} u}{\partial_{r} u} \\
& \boldsymbol{Z}=\frac{r \operatorname{sen} p \operatorname{sen} q \partial_{r} u-\cos p \operatorname{sen} q \partial_{\rho} u-\frac{\cos q}{\operatorname{sen} p} \partial_{q} u}{\partial_{r} u}, \\
& \boldsymbol{R}= \pm \frac{\sqrt{ }\left\{\boldsymbol{r}^{2}\left(\partial_{r} u\right)^{2}+\left(\partial_{p} u\right)^{2}+\left(\partial_{q} u\right)^{2}\right\}}{\partial_{r} u} .
\end{aligned}
$$

Se l'equazione polare della superficie sia risoluta rapporto ad $r$, allora, come già si è veduto per le superficie del sistema positivo, si ricaverà

$$
\begin{aligned}
& X=r \cos p-r^{\prime} \operatorname{sen} p, \quad Y=r \operatorname{sen} p \cos q+r^{\prime} \cos p \cos q-\frac{r_{1} \operatorname{sen} q}{\operatorname{sen} p}, \\
& Z=r \operatorname{sen} p \operatorname{sen} q+r^{\prime} \cos p \operatorname{sen} q+\frac{r_{1} \cos q}{\operatorname{sen} p}, \quad \boldsymbol{R}= \pm \sqrt{ }\left(r^{2}+r^{\prime 2}+r_{1}^{2}\right) .
\end{aligned}
$$

Reciprocamente i valori delle coordinate $x, y, z$, \& del raggio $r$ si potrebbero esprimere per le coordinate polari della nuova superficie con formole del tutto somiglianti a quelle che termina il parag. $6^{\circ}$.

$10^{\circ}$. Terminiamo questa prima parte dell' addizzione col dimostrare l'eguaglianza degli angoli formati dai piani tangenti con i rispettivi raggi vettori nel doppio sistema positivo, o negativo. Sia $\alpha$ l'angolo formato dal piano tangente la superficie curvia $u=0$ nel punto $(x, y, z)$ con il raggio $r$ condotto dall' origine: abbassando dalla stessa origine una perpendicolare $\boldsymbol{R}$ sopra 
la direzione del piano tangente, si avrà

$$
\boldsymbol{R}=\boldsymbol{r} \operatorname{sen} \alpha \quad 0, \quad \operatorname{sen} \alpha=\frac{\boldsymbol{R}}{\boldsymbol{r}} ;
$$

quindi supposto l'equazione della superficie risoluta rapporto a $z$, e fatto per le derivate parziali

$$
p=\frac{d z}{d x}, \quad q=\frac{d z}{d y},
$$

avremo dai valori di $\boldsymbol{R}$, ed $\boldsymbol{r}$ dalle formole del parag. $3^{\circ}$ :

$$
\operatorname{sen} \alpha=\frac{p x+q y-z}{\sqrt{\left(x^{2}+y^{2}+z^{2}\right) \sqrt{ }\left(1+p^{2}+q^{2}\right)}} .
$$

L'estremità della perpendicolare $\boldsymbol{R}$ appartiene a un punto $(\boldsymbol{X}, \boldsymbol{Y}, \boldsymbol{Z})$ della superficie derivata, e perciò chiamando $\alpha^{\prime}$ l'angolo, formato dal piano tangente la superficie nello stesso punto $(\boldsymbol{X}, \boldsymbol{Y}, \boldsymbol{Z})$ con il raggio $\boldsymbol{R}$, e facendo per le derivate parziali

si avrà egualmente

$$
\boldsymbol{P}=\frac{d Z}{d X}, \quad \boldsymbol{Q}=\frac{d Z}{d Y}
$$

$$
\operatorname{sen} \alpha^{\prime}=\frac{P X+Q Y-Z}{\sqrt{ }\left(X^{2}+Y^{2}+Z^{2}\right) \sqrt{ }\left(1+\bar{P}^{2}+Q^{2}\right)} .
$$

Per conoscere la relazione che passa fra gli angoli $\alpha, \alpha^{\prime}$, facciamo una supposizione, la quale nulla alterando la generalità della questione, ci potrà nello stesso tempo far giungere con la massima facilità alla medesima conclusione. Riprendiamo dal parag. $3^{\circ}$ i valori di $\boldsymbol{X}, \boldsymbol{Y}, \boldsymbol{Z}$, ciò̀

$$
\boldsymbol{X}=\frac{p(p x+q y-z)}{1+p^{2}+q^{2}}, \quad \boldsymbol{Y}=\frac{q(p x+q y-z)}{1+p^{2}+q^{2}}, \quad \boldsymbol{Z}=\frac{z-p x-q y}{1+p^{2}+q^{2}},
$$

e scegliamo il piano $x y$ parallelo al piano tangente, sarà $p=0, q=0$, d'onde $\boldsymbol{X}=\mathbf{0}, \boldsymbol{Y}=0, \boldsymbol{Z}=\boldsymbol{z}$ per cui

$$
\operatorname{sen} \alpha=-\frac{z}{\sqrt{\left(x^{2}+y^{2}+z^{2}\right)}}, \quad \operatorname{sen} \alpha^{\prime}=-\frac{1}{\sqrt{\left(1+P^{2}+Q^{2}\right)}} .
$$

Pongasi in fine per le derivate parziali del secondo ordine

$$
r=\frac{d^{2} z}{d x^{2}}, \quad s=\frac{d^{2} z}{d x d y}, \quad t=\frac{d^{2} z}{d y^{2}},
$$

le quali potranno ritenere un valor finito quantunque sia $p=0, q=0$. Ció posto, indichiamo per $\boldsymbol{X}^{\prime}, \boldsymbol{X}_{1}, \ldots$ le derivate parziali rapporto ad $x$, ed $\boldsymbol{y}$ delle variabili $\boldsymbol{X}, \boldsymbol{Y}, \boldsymbol{Z}$, è noto dalle trasformazioni dei differenziali parziali che le derivate $\boldsymbol{P}, \boldsymbol{Q}$ si esprimono per

$$
P=\frac{X^{\prime} Z_{1}-X_{1} Z^{\prime}}{X^{\prime} Y_{1}-Y^{\prime} X_{1}}, \quad Q=\frac{Y_{1} Z^{\prime}-Z_{1} Y^{\prime}}{X^{\prime} Y_{1}-Y^{\prime} X_{1}}
$$


Ora dai valori di $X, Y, Z$ si ottiene col fare dopo le derivazioni $p=0, q=0$ :

dalle quali'

$$
\begin{aligned}
X^{\prime}=-r z, \quad X_{1}=-s z, & Y^{\prime}=-s z, \quad Y_{1}=-z t, \\
Z^{\prime}=-(r x+s y), & Z_{1}=-(t y+s x),
\end{aligned}
$$

$$
\begin{aligned}
X^{\prime} Y_{1}-X_{1} Y^{\prime}= & z^{2}\left(r t-s^{2}\right), \quad X^{\prime} Z_{1}-X_{1} Z^{\prime}=z y\left(r t-s^{2}\right), \\
& Y_{1} Z^{\prime}-Y^{\prime} Z_{1}=x \approx\left(r t-s^{2}\right),
\end{aligned}
$$

e perciò

$$
\sqrt{ }\left(1+\boldsymbol{P}^{2}+\boldsymbol{Q}^{2}\right)=\frac{\sqrt{ }\left(x^{2}+y^{2}+z^{2}\right)}{z}
$$

Da ciò ne segue immediatamente

$$
\operatorname{sen} \alpha=\operatorname{sen} \alpha^{\prime}, \quad \text { ed anche } \alpha=\alpha^{\prime} \text {. }
$$

Il Sigr Roberts nella citala Memorià è giunto per una dimostrazione geometrica semplicissima a riconoscere l'eguaglianza degli angoli formati dalle tangenti con i respettivi raggi vettori nelle curve del doppio sistema positivo, e negativo: aggiungiamo di più che il ragionamento del Sigr ${ }^{r}$ Roberts si estende ancora alle superficie curve.

11 ${ }^{\circ}$. Presentiamo ora delle applicazioni delle precedenti formole alla quadratura di una qualche superficie. $\grave{\mathbf{E}}$ noto che la quadratura delle superficie curve, considerando una delle cóordinate $\boldsymbol{Z}$ come funzione delle due altre $\boldsymbol{X}, \boldsymbol{Y}$, dipende "dall' integrale dụplicato

$$
S=\iint d X d Y \sqrt{ } \boldsymbol{S}\left(1+\left(\frac{d Z}{d X}\right)^{2}+\left(\frac{d Z}{d Y}\right)^{2}\right\}
$$

Se le $\boldsymbol{X}, \boldsymbol{Y}, \boldsymbol{Z}$ sieno funzioni di due nuove variabili $p, q$, e rappresentando per $\boldsymbol{X}^{\prime}, \boldsymbol{Y}^{\prime}, \boldsymbol{Z}^{\prime}$ le derivate parziali delle $\boldsymbol{X}, \boldsymbol{Y}, \boldsymbol{Z}$ rapporto a $p$, e per $\boldsymbol{X}_{1}, \boldsymbol{Y}_{1}, \boldsymbol{Z}_{1}$ le derivate delle medesime rapporto a $q$, l'integrale relativamente alle nuove variabili $p, q$ si trasformerà in

ove per brevità

$$
\boldsymbol{S}=\iint d \boldsymbol{p} d \boldsymbol{q} V\left(\boldsymbol{U}^{2}+\boldsymbol{V}^{2}+\boldsymbol{W}^{2}\right)
$$

$$
U=X^{\prime} Y_{1}-X_{1} Y^{\prime}, \quad V=X_{1} Z^{\prime}-X^{\prime} Z_{1}, \quad W=Y^{\prime} Z_{1}-Y_{1} Z^{\prime}
$$

Queste trasformazioni già conosciute dai geometri, si trovano fra gli altri, per le, formole della quadratura, e cubatura nella Teoria delle funzioni di Lagrange. Supponiamo adunque che si voglia conoscere la quadratura della superficie curva negativa proveniente dal centro dell' ellissoide; se per maggior semplicità si prenda per l'equazione dell' ellissoide

$$
\frac{x^{2}}{a}+\frac{y^{2}}{b}+\frac{z^{2}}{c}=1
$$


noi avremo dalle formole del parag. $8^{\circ}$ per le coordinate $\boldsymbol{X}, \boldsymbol{Y}, \boldsymbol{Z}$ della superficie derivata:

$$
\begin{aligned}
& X=\frac{u}{\sqrt{a}}\left(a u^{2}+(2 a-b) v^{2}+(2 a-c) w^{2}\right) \\
& Y=\frac{v}{\sqrt{b}}\left(b v^{2}+(2 b-a) u^{2}+(2 b-c) w^{2}\right) \\
& Z=\frac{w}{\sqrt{c}}\left(c w^{2}+(2 c-a) u^{2}+(2 c-b) v^{2}\right)
\end{aligned}
$$

Le variabili $u, v, w$, sono le coordinate sferiche

$$
u=\cos p, \quad v=\operatorname{sen} p \cos q, \quad w=\operatorname{sen} p \operatorname{sen} q .
$$

Facendo le derivazioni rapporto a $p$, avremo dopo la riduzione dei termini per le potenze di $\operatorname{sen} p$, senq:

$$
\begin{aligned}
& X^{\prime}=\frac{\operatorname{sen} p}{\sqrt{ } a}\left(a-2 b+3(b-a) \operatorname{sen}^{2} p-2(c-b) \operatorname{sen}^{2} q+3(c-b) \operatorname{sen}^{2} p \operatorname{sen}^{2} q\right), \\
& Y^{\prime}=\frac{\cos p \cos q}{\sqrt{ } b}\left(2 b-a+3(a-b) \operatorname{sen}^{2} p+3(b-c) \operatorname{sen}^{2} p \operatorname{sen}^{2} q\right), \\
& Z^{\prime}=\frac{\cos p \operatorname{sen} q}{\sqrt{ } c}\left(2 c-a+3(a-b) \operatorname{sen}^{2} p+3(b-c) \operatorname{sen}^{2} p \operatorname{sen}^{2} q\right) .
\end{aligned}
$$

Nella stessa guisa derivando rapporto a $q$, otteniamo

$$
\begin{aligned}
& X_{1}=\frac{2(b-c)}{\sqrt{ } a} \operatorname{sen}^{2} p \cos p \operatorname{sen} q \cos q \\
& Y_{1}=\frac{\operatorname{sen} p \operatorname{sen} q}{\sqrt{ } b}\left(a-2 b+(3 b-a-2 c) \operatorname{sen}^{2} p+3(c-b) \operatorname{sen}^{2} p \operatorname{sen}^{2} q\right), \\
& Z_{1}=\frac{\operatorname{sen} p \cos q}{\sqrt{ } c}\left(2 c-a+(a-b) \operatorname{sen}^{2} p-3(c-b) \operatorname{sen}^{2} p \operatorname{sen}^{2} q\right)
\end{aligned}
$$

Formando con queste derivate le differenze dei prodotti indicate da $\boldsymbol{U}, \boldsymbol{V}, \boldsymbol{W}$, e ponendo ancora

$$
\begin{aligned}
\boldsymbol{R}= & (a-2 b)(a-2 c)+2(b-a)(2 a-b-3 c) \operatorname{sen}^{2} p \\
& -2\left(c^{2}-b^{2}\right) \operatorname{sen}^{2} p \operatorname{sen}^{2} q+3(b-a) \operatorname{sen}^{4} p \\
& +6(b-a)(c-b) \operatorname{sen}^{4} p \operatorname{sen}^{2} q+3(c-b)^{2} \operatorname{sen}^{4} p \operatorname{sen}^{4} q
\end{aligned}
$$

otterremo doppo tutte le richieste riduzioni:

$$
U=\frac{\sqrt{ } c \cdot R \operatorname{sen}^{2} p \operatorname{sen} q}{\sqrt{ }(a b c)}, \quad V=\frac{\sqrt{ } b \cdot R \operatorname{sen}^{2} p \cos q}{\sqrt{ }(a b c)}, \quad W=\frac{\sqrt{ } a \cdot R \operatorname{sen} p \cos p}{\sqrt{ }(a b c)},
$$

d'onde nuovamente per i valori di $u, v, w$ :

$$
\sqrt{ }\left(\boldsymbol{U}^{2}+\boldsymbol{V}^{2}+\boldsymbol{W}^{2}\right)=\frac{\boldsymbol{R} \operatorname{sen} p}{\sqrt{ }(a b c)} \sqrt{ }\left(a u^{2}+b v^{2}+c w^{2}\right)
$$

e perciò la quadratura della superficie dipenderà dall' integrale duplicato

$$
S=\frac{1}{\sqrt{ }(a b c)} \iint \boldsymbol{R} \operatorname{sen} p d p d q \sqrt{ }\left(a u^{2}+b v^{2}+c w^{2}\right)
$$


Integrando fra $i$ limiti $p=0, p=\frac{1}{2} \pi, q=0, q=\frac{1}{2} \pi$, si ottiene l'ottava parte della superficie, per cui la quadratura dell' intera superficie sarà espressa per l'integrale definito duplicato

$$
S=\frac{8}{\sqrt{(a b c)}} \int_{0}^{\frac{1}{b} \pi} \int_{0}^{\frac{1}{2} \pi} R \operatorname{sen} p d p d q \sqrt{ }\left(a u^{2}+b v^{2}+c w^{2}\right) .
$$

Tutto adunque consiste a ridurre questo integrale duplicato ad un integrale semplice, ciò che noi verremo successivamente ad esporre.

$12^{\circ}$. Ripreso primieramente il valore di $\boldsymbol{R}$, si sostituisca nel secondo nel quarto, e nel quinto termine

$$
\begin{gathered}
\operatorname{sen}^{2} p=1-\cos ^{2} p, \quad \operatorname{sen}^{4} p=1-2 \cos ^{2} p+\cos ^{4} p, \\
\operatorname{sen}^{4} p=\operatorname{sen}^{2} p\left(1-\cos ^{2} p\right),
\end{gathered}
$$

si ricavera facilmente per $\mathrm{i}$ valori di $u, v, w$ :

$$
\begin{aligned}
\boldsymbol{R}= & (b-2 a)(b-2 c)-2(b-a)(2 b-a-3 c) u^{2} \\
& +2(c-b)(2 b-c-3 a) w^{2}+3(b-a)^{2} u^{4} \\
& -6(b-a)(c-b) u^{2} w^{2}+3(c-b)^{2} w^{4} .
\end{aligned}
$$

Una prima integrazione si eseguisce più agevolmente cọl sostituire due nuove variabili $\theta, \omega$ invece di $p, q$ ed atte a togliere le irrazionalità. Pongasi

$$
\xi=\cos \theta, \quad \eta=\operatorname{sen} \theta \cos \omega, \quad \zeta=\operatorname{sen} \theta \operatorname{sen} \omega,
$$

e supponiamo che fra gli angoli $p, q, \theta, \omega$ sussistano le relazioni

$\xi=\frac{\sqrt{ } a \cdot u}{\sqrt{ }\left(a u^{2}+b v^{2}+c w^{2}\right)}, \quad \eta=\frac{\sqrt{ } b \cdot v}{\sqrt{\left(a u^{2}+b v^{2}+c w^{2}\right)}}, \quad \zeta=\frac{\sqrt{c} \cdot v}{\sqrt{\left(a u^{2}+b v^{2}+c w^{2}\right)}}$.

Di questa identica sostituzione ho fatto già uso nella mia precedente Memoria per altre quadrature, e cubature. Dai valori di $\xi, \eta, \zeta$ si ricavano reciprocamente quei di $u, v, v$; cosicchè facendo

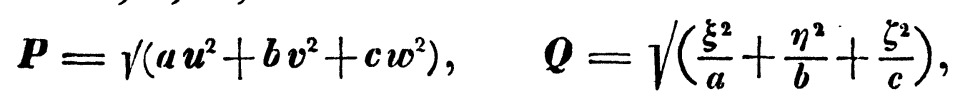

si hà non solamente $\boldsymbol{P Q}=1$, mà ben anche

$$
u=\frac{\xi}{\sqrt{a . Q}}, \quad v=\frac{\eta}{\sqrt{b . Q}}, \quad u=\frac{\zeta}{\sqrt{c . Q}} .
$$

La prima di queste formole è lo stesso che .

$$
\cos p=\frac{\cos \theta}{\sqrt{ } a \cdot \sqrt{\left(\frac{\cos ^{2} \theta}{a}+\frac{\operatorname{sen}^{2} \theta \cos ^{2} \omega}{b}+\frac{\operatorname{sen}^{2} \theta \operatorname{sen}^{2} \omega}{c}\right)}},
$$

e le altre due dalla divisione di $w$ per $v$ porgono

$$
\operatorname{tang} q=\frac{\sqrt{ } b}{\sqrt{ } c} \operatorname{tang} \omega,
$$


ove ai limili stabiliti per $p$, e $q$ corrispondono i medesimi limiti per $\theta$, ed $\omega$. L'elemento differenziale relativo ai nuovi angoli $\theta, \omega$, che si ha da sostituire all'elemento $\operatorname{sen} p \partial p \partial q$, si trova col differenziare $\cos p$ nella supposizione di $q$ costante, ossia di $\omega$ costante, ciò che porge

$$
\begin{aligned}
\operatorname{sen} p \partial p & =\frac{\left(b \operatorname{sen}^{2} \omega+c \cos ^{2} \omega\right) \operatorname{sen} \theta \partial \theta}{b c \cdot \sqrt{ } a \cdot \sqrt{\left(\frac{\cos ^{2} \theta}{a}+\frac{\operatorname{sen}^{2} \theta \cos ^{2} \omega}{b}+\frac{\operatorname{sen}^{2} \theta \operatorname{sen}^{2} \omega}{c}\right)^{3}}} \\
\partial q & =\frac{\sqrt{ }(b c) \partial q}{\left(b \operatorname{sen}^{2} \omega+c \cos ^{2} \omega\right)} .
\end{aligned}
$$

Quindi all' elemento $\operatorname{sen} p \partial p \partial q$ si dovrà sostituire il nuovo elemento

$$
\frac{\operatorname{sen} \theta \partial \theta \partial \omega}{\sqrt{(a b c) Q^{3}}}
$$

Elevando la $\boldsymbol{Q}$ al quadrato, abbiamo

$$
Q^{2}=\frac{b c \xi^{2}+a c \eta^{2}+a b \xi^{2}}{a b c}
$$

d'onde sostituendo nel valore di $\boldsymbol{R}$ i valori di $u, v, w$ espressi per $\xi, \eta, \zeta$, e facendo per brevità

$$
\begin{array}{ll}
\boldsymbol{A}=b^{2} c^{2}(a-2 b)(a-2 c), & \boldsymbol{B}=a^{2} c^{2}(b-2 a)(b-2 c), \\
C=a^{2} b^{2}(c-2 a)(c-2 b), & \boldsymbol{A}^{\prime}=a b c^{2}\left(a b+a c+b c-a^{2}-b^{2}\right), \\
\boldsymbol{B}^{\prime}=a c b^{2}\left(a b+a c+b c-a^{2}-c^{2}\right), & C^{\prime}=b c a^{2}\left(a b+a c+b c-b^{2}-c^{2}\right),
\end{array}
$$

si ricaverà

$$
\boldsymbol{R}=\frac{A \xi^{4}+B \eta^{4}+C \zeta^{4}+2 A^{\prime} \xi^{2} \eta^{2}+2 B^{\prime} \xi^{2} \zeta^{2}+2 C^{\prime} \eta^{2} \zeta^{2}}{\left(b c \xi^{2}+a c \eta^{2}+a b \zeta^{2}\right)^{2}}
$$

Pongasi per il numeratore

$$
\boldsymbol{R}_{1}=\boldsymbol{A} \xi^{4}+\boldsymbol{B} \eta^{4}+\boldsymbol{C} \zeta^{4}+2 \boldsymbol{A}^{\prime} \xi^{2} \eta^{2}+2 \boldsymbol{B}^{\prime} \xi^{2} \zeta^{2}+2 \boldsymbol{C}^{\prime \prime} \eta^{2} \zeta^{2},
$$

allora il valore di $\boldsymbol{S}$ dato dall' ultima formola dell' antecedente parag ${ }^{0}$ diverrà

$$
\boldsymbol{S}=8 a b c \int_{0}^{\frac{1}{3} \pi} \int_{0}^{\frac{1}{2} \pi} \frac{\boldsymbol{R}_{1} \operatorname{sen} \theta \partial \theta \partial \omega}{\left(b c \cos ^{2} \theta+a c \operatorname{sen}^{2} \theta \cos ^{2} \omega+a b \operatorname{sen}^{2} \theta \operatorname{sen}^{2} \omega\right)^{4}} .
$$

Tal'è il nuovo integrale definito privo d'irrazionalità.

13. Una prima integrazione definita fra gli indicati limiti si eseguisce facilmente rapporto alla variabile $\omega$. Per semplificare le operazioni analitiche, sia

$$
\alpha^{2}=c\left(b \cos ^{2} \theta+a \operatorname{sen}^{2} \theta\right), \quad \beta^{2}=b\left(c \cos ^{2} \theta+a \operatorname{sen}^{2} \theta\right),
$$

ed insieme

Crelle's Journal f. d. M. Bd. XXXIV. Heft 2. 


$$
\begin{array}{ll}
\boldsymbol{V}=\int_{0}^{\frac{1}{2} \pi} \frac{\partial \omega}{\left(\alpha^{2} \cos ^{2} \omega+\beta^{2} \operatorname{sen}^{2} \omega\right)^{4}}, & V_{1}=\int_{0}^{\frac{1}{2} \pi} \frac{\cos ^{4} \omega \partial \omega}{\left(\alpha^{2} \cos ^{2} \omega+\beta^{2} \operatorname{sen}^{2} \omega\right)^{4}}, \\
V_{2}=\int_{0}^{\frac{1}{2} \pi} \frac{\operatorname{sen}^{4} \omega \partial \omega}{\left(\alpha^{2} \cos ^{2} \omega+\beta^{2} \operatorname{sen}^{2} \omega\right)^{4}}, & V_{3}=\int_{0}^{\frac{1}{2} \pi} \frac{\cos ^{2} \omega \partial \omega}{\left(\alpha^{2} \cos ^{2} \omega+\beta^{2} \operatorname{sen}^{2} \omega\right)^{4}}, \\
V_{4}=\int_{0}^{\frac{1}{2} \pi} \frac{\operatorname{sen}^{2} \omega \partial \omega}{\left(\alpha^{2} \cos ^{2} \omega+\beta^{2} \operatorname{sen}^{2} \omega\right)^{4}}, & V_{5}=\int_{0}^{\frac{1}{2} \pi} \frac{\operatorname{sen}^{2} \omega \cos ^{2} \omega \partial \omega}{\left(\alpha^{2} \cos ^{2} \omega+\beta^{2} \operatorname{sen}^{2} \omega\right)^{4}},
\end{array}
$$

L'integrale definito duplicato dopo la sostituzione dei valori di $\boldsymbol{V}, \boldsymbol{V}_{1}, \boldsymbol{V}_{2}, \ldots$ che verremo a determinare sarà ridotto ad una somma d'integrali definiti semplici. È facile di poter conoscere la forma di questi ultimi integrali definiti. Pongasi

$$
\begin{array}{ll}
\boldsymbol{S}=\int_{U}^{\frac{1}{3} \pi} V \cos ^{4} \theta \operatorname{sen} \theta \partial \theta, & \boldsymbol{S}_{1}=\int_{U}^{\frac{1}{3} \pi} V_{1} \operatorname{sen}^{5} \theta \partial \theta, \\
\boldsymbol{S}_{2}=\int_{U}^{\frac{1}{2} \pi} V_{2} \operatorname{sen}^{5} \theta \partial \theta, & \boldsymbol{S}_{3}=\int_{0}^{\frac{1}{3} \pi} V_{3} \cos ^{2} \theta \operatorname{sen}^{3} \theta \partial \theta, \\
\boldsymbol{S}_{4}=\int_{U}^{\frac{1}{2} \pi} V_{4} \cos ^{2} \theta \operatorname{sen}^{3} \theta \partial \theta, & \boldsymbol{S}_{5}=\int_{0}^{\frac{1}{2} \pi} V_{5} \operatorname{sen}^{5} \theta \partial \theta,
\end{array}
$$

il valore di $\boldsymbol{S}$ si presenterà sotto la forma

$$
\boldsymbol{S}=8 a b c\left(A \boldsymbol{S}_{0}+B \boldsymbol{S}_{1}+C \boldsymbol{S}_{2}+2 A^{\prime} \boldsymbol{S}_{3}+2 B^{\prime} \boldsymbol{S}_{4}+2 C^{\prime} \boldsymbol{S}_{5}\right) \text {. }
$$

Veniamo alla determinazione degli integrali definiti $\boldsymbol{V}, \boldsymbol{V}_{1}, \boldsymbol{V}_{2}, \ldots$ A questo proposito, osserviamo che sostituendo $\approx=\beta$ tang $\omega$, si ottiene immediatamente

$$
\int_{0}^{\frac{1}{2} \pi} \frac{\partial \omega}{\alpha^{2} \cos ^{2} \omega+\beta^{2} \operatorname{sen}^{2} \omega}=\frac{1}{2} \pi \cdot \frac{1}{\alpha \beta} \text {. }
$$

Coll' eseguire delle derivazioni rapporto alle costanti $\alpha, \beta$, potremo giungere a diversi altri integrali definiti, fra $i$ quali si troveranno quei che ci occorrono. Cosi da una prima derivazione rapporto ad $\alpha$, e $\beta$ deduciamo dopo la divisione o per $2 \alpha$, o per $2 \beta$ :

$\int_{0}^{\frac{1}{b} \pi} \frac{\cos ^{2} \omega \partial \omega}{\left(\alpha^{2} \cos ^{2} \omega+\beta^{2} \operatorname{sen}^{2} \omega\right)^{2}}=\frac{1}{4} \pi \cdot \frac{1}{\alpha^{3} \beta}, \quad \int_{0}^{\frac{1}{2} \pi} \frac{\operatorname{sen}^{2} \omega \partial \omega}{\left(\alpha^{2} \cos ^{2} \omega+\beta^{2} \operatorname{sen}^{2} \omega\right)^{2}}=\frac{1}{4} \pi \cdot \frac{1}{\alpha \beta^{3}}$ quindi da lor somma

$$
\int_{0}^{\frac{1}{\pi} \pi} \frac{\partial \omega}{\left(\alpha^{2} \cos ^{2} \omega+\beta^{2} \operatorname{sen}^{2} \omega\right)^{2}}=\pi \pi\left(\frac{1}{\alpha \beta^{3}}+\frac{1}{\beta \alpha^{3}}\right) .
$$

Questo integrale fủ riportato senza dimostrazione al parag. $7^{\circ}$ della mia citata Memoria. Proseguiamo nel nuovo ultimo integrale la derivazione rapporto alle costanti $\alpha, \beta$, avremo egualmente 


$$
\begin{aligned}
& \text { 1. } \int_{0}^{\frac{1}{2} \pi} \frac{\cos ^{2} \omega \partial \omega}{\left(\alpha^{2} \cos ^{2} \omega+\beta^{2} \operatorname{sen}^{2} \omega\right)^{3}}=\frac{1}{1^{1}} \pi\left(\frac{3}{\beta \alpha^{5}}+\frac{1}{\alpha^{3} \beta^{3}}\right), \\
& \text { 2. } \int_{0}^{\frac{1}{2} \pi} \frac{\operatorname{sen}^{2} \omega \partial \omega}{\left(\alpha^{2} \cos ^{2} \omega+\beta^{2} \operatorname{sen}^{2} \omega\right)^{3}}=\frac{1}{1^{6}} \pi\left(\frac{3}{\alpha \beta^{5}}+\frac{1}{\alpha^{3} \beta^{3}}\right),
\end{aligned}
$$

d'onde, sommando,

$$
\int_{0}^{\frac{1}{2} \pi} \frac{\partial \omega}{\left(\alpha^{2} \cos ^{2} \omega+\beta^{2} \operatorname{sen}^{2} \omega\right)^{3}}=\frac{1}{16} \pi\left(\frac{3}{\alpha \beta^{5}}+\frac{3}{\beta \alpha^{5}}+\frac{2}{\alpha^{3} \beta^{3}}\right) .
$$

Questo integrale si presentò ancora nel parag. $8^{\circ}$ della mia Memoria in alcune speculazioni geometriche della stessa specie. Nel nuovo integrale definito si prosegua la derivazione rapporto alle consuete costanti, si avrà

$$
\begin{aligned}
& V_{4}=\int_{0}^{\frac{1}{2} \pi} \frac{\operatorname{sen}^{2} \omega \partial \omega}{\left(\alpha^{2} \cos ^{2} \omega+\beta^{2} \operatorname{sen}^{2} \omega\right)^{4}}=\frac{1}{3} \pi\left(\frac{5}{\alpha \beta^{7}}+\frac{1}{\alpha^{5} \beta^{3}}+\frac{6}{\alpha^{3} \beta^{5}}\right), \\
& V_{3}=\int_{0}^{\frac{1}{3} \pi} \frac{\cos ^{2} \omega \partial \omega}{\left(\alpha^{2} \cos ^{2} \omega+\beta^{2} \operatorname{sen}^{2} \omega\right)^{4}}=\frac{1}{3} \pi\left(\frac{1}{\alpha^{3} \beta^{5}}+\frac{5}{\beta \alpha^{7}}+\frac{6}{\beta^{3} \alpha^{5}}\right),
\end{aligned}
$$

quali sommate porgeranno

$$
V=\int_{0}^{\frac{1}{2} \pi} \frac{\partial \omega}{\left(\alpha^{3} \cos ^{2} \omega+\beta^{2} \operatorname{sen}^{2} \omega\right)^{4}}=\frac{1}{3} \pi\left(\frac{5}{\alpha \beta^{7}}+\frac{5}{\beta \alpha^{7}}+\frac{7}{\alpha^{3} \beta^{3}}+\frac{7}{\beta^{3} \alpha^{5}}\right) .
$$

Nello stesso modo eseguendo una derivazione nelle formole (1. e 2.) rapporto ad $\alpha$, e $\beta$, si ricaverà dopo la divisione per $6 \alpha$, o per $6 \beta$,

$$
\begin{aligned}
& V_{1}=\int_{0}^{\frac{1}{2} \pi} \frac{\cos ^{4} \omega \partial \omega}{\left(\alpha^{2} \cos ^{2} \omega+\beta^{2} \operatorname{sen}^{2} \omega\right)^{4}}=\frac{1}{3} \pi\left(\frac{5}{\beta \alpha^{7}}+\frac{1}{\alpha^{3} \beta^{3}}\right), \\
& V_{2}=\int_{0}^{\frac{1}{2} \pi} \frac{\operatorname{sen}^{4} \omega \partial \omega}{\left(\alpha^{2} \cos ^{2} \omega+\beta^{2} \operatorname{sen}^{2} \omega\right)^{4}}=\frac{1}{3} \pi\left(\frac{5}{\alpha \beta^{7}}+\frac{1}{\alpha^{3} \beta^{5}}\right) .
\end{aligned}
$$

In fine derivando la (1.) rapporto a $\beta$, otterremo

$$
V_{5}=\int_{0}^{\frac{1}{2} \pi} \frac{\operatorname{sen}^{2} \omega \cos ^{2} \omega \partial \omega}{\left(\alpha^{2} \cos ^{2} \omega+\beta^{2} \operatorname{sen}^{2} \omega\right)^{4}}=\frac{1}{3} \pi\left(\frac{1}{\alpha^{5} \beta^{3}}+\frac{1}{\alpha^{3} \beta^{5}}\right)
$$

Dopo di aver calcolato gli integrali $V, V_{1}, V_{2}, \ldots$ che sono funzioni della sola variabile $\theta$, restano a calcolarsi gli altri integrali espressi per $\boldsymbol{S}_{11}, \boldsymbol{S}_{1}, \boldsymbol{S}_{2}, \ldots$; quali dipenderanno tutti dai trascendenti ellittici di prima e seconda specie. Per meglio scorgere la natura di queste altre riduzioni, facciamo un cangiamento della variabile $\theta$ in una nuova variabile. Supponiamo, per fissar le idee, $a<b<c$, e sia

$$
\cos \mu=\frac{\sqrt{ } a}{\sqrt{ } c}, \quad \cos \nu=\frac{\sqrt{ } a}{\sqrt{b}}, \quad k^{2}=1-\frac{\operatorname{tang}^{2} \nu}{\operatorname{tang}^{2} \mu}=\frac{c-b}{c-a},
$$


quindi denotato per $\varphi$ un'angolo variabile, pongasi

per cui

$$
\cos \theta=\cot \mu \operatorname{tang} \varphi=\frac{\sqrt{ } a}{\sqrt{(c-a)}} \operatorname{tang} \varphi,
$$

$\cos \theta=\frac{\sqrt{ } a}{\sqrt{(c-a)}} \cdot \frac{\operatorname{sen} \varphi}{\cos \varphi}, \quad \operatorname{sen} \theta=\frac{\sqrt{ }\left(c \cos ^{2} \varphi-a\right)}{\sqrt{ }(c-a) \cos \varphi}, \operatorname{sen} \theta \partial \theta=-\frac{\sqrt{ } a}{\sqrt{(c-a)}} \cdot \frac{\partial \varphi}{\cos ^{2} \varphi}$. Ai limiti $\theta=0, \theta=\frac{1}{2} \pi$ corrispondono $\varphi=\mu, \varphi=0$, e perciò gli integrali definiti fra i limiti $\varphi=\mu, \varphi=0$, saranno eguali agli integrali definiti fra i limiti $\varphi=0, \varphi=\mu$, purché si faccia un cangiamento di segni: la quantilà $k$ sarà $<1$, e percio, fatto

i valori di $\alpha^{0}$, e $\beta^{0}$ diverranno

$$
\Delta=\sqrt{ }\left(1-k^{2} \operatorname{sen}^{2} \varphi\right)
$$

$$
\alpha=\frac{\sqrt{ }(a c) \Delta}{\cos \varphi}, \quad \beta=\frac{\sqrt{ }(a b)}{\cos \varphi} .
$$

Di qui per le quantità $V, V_{1}, V_{2}, \ldots$ si hà

$$
\begin{aligned}
& V=\frac{\pi \cos ^{8} \varphi}{32 a^{4} b^{3} c^{3} \sqrt{ }(b c)}\left(\frac{5 c^{3}}{\Delta}+\frac{7 b c^{2}}{\Delta^{3}}+\frac{7 c b^{2}}{\Delta^{5}}+\frac{5 b^{3}}{\Delta^{7}}\right) \\
& V_{1}=\frac{\pi \cos ^{8} \varphi}{32 a^{4} c^{3} b \sqrt{ }(b c)}\left(\frac{c}{\Delta^{3}}+\frac{5 b}{\Delta^{7}}\right) \\
& V_{2}=\frac{\pi \cos ^{8} \varphi}{32 a^{4} b^{3} c \sqrt{ }(b c)}\left(\frac{5 c}{\Delta}+\frac{b}{\Delta^{3}}\right) \\
& V_{3}=\frac{\pi \cos ^{8} \varphi}{32 a^{4} c^{3} b^{2} \sqrt{ }(b c)}\left(\frac{c^{2}}{\Delta^{3}}+\frac{6 b c}{\Delta^{5}}+\frac{5 b^{2}}{\Delta^{7}}\right) \\
& V_{4}=\frac{\pi \cos ^{8} \varphi}{32 a^{4} b^{3} c^{2} \sqrt{(b c)}}\left(\frac{5 c^{2}}{\Delta}+\frac{6 b c}{\Delta^{3}}+\frac{b^{2}}{\Delta^{5}}\right) \\
& V_{5}=\frac{\pi \cos ^{6} \varphi}{32 a^{4} b^{2} c^{2} \sqrt{ }(b c)}\left(\frac{c}{\Delta^{3}}+\frac{b}{\Delta^{3}}\right) .
\end{aligned}
$$

Infine fatto per brevità

$$
m=\frac{\pi}{32 a b c \sqrt{ }(a b c) \sqrt{ }(c-a)^{3}},
$$

i valori degli integrali definiti $\boldsymbol{S}_{0}, \boldsymbol{S}_{1}, \boldsymbol{S}_{2}, \ldots$ saranno

$$
\begin{aligned}
& S_{0}=\frac{m}{b^{2} c^{2}} \int_{0}^{\mu}\left(\frac{5 c^{3}}{\Delta}+\frac{7 b c^{2}}{\Delta^{3}}+\frac{7 c b^{2}}{\Delta^{3}}+\frac{5 b^{3}}{\Delta^{7}}\right) \cos ^{2} \varphi \operatorname{sen}^{4} \varphi \partial \varphi \\
& S_{1}=\frac{m}{a^{2} c^{2}} \int_{0}^{\mu}\left(\frac{c}{\Delta^{3}}+\frac{5 b}{\Delta^{7}}\right)\left(c \cos ^{2} \varphi-a\right)^{2} \cos ^{2} \varphi \partial \varphi \\
& S_{2}=\frac{m}{a^{2} b^{2}} \int_{0}^{\mu}\left(\frac{5 c}{\Delta}+\frac{b}{\Delta^{3}}\right)\left(c \cos ^{2} \varphi-a\right)^{2} \cos ^{2} \varphi \partial \varphi
\end{aligned}
$$




$$
\begin{aligned}
& S_{3}=\frac{m}{a b c^{2}} \int_{0}^{\mu}\left(\frac{c^{2}}{\Delta^{3}}+\frac{6 b c}{\Delta^{5}}+\frac{5 b^{2}}{\Delta^{2}}\right)\left(c \cos ^{2} \varphi-a\right) \operatorname{sen}^{2} \varphi \cos ^{2} \varphi \partial \varphi, \\
& S_{4}=\frac{m}{a c b^{2}} \int_{0}^{\mu}\left(\frac{5 c^{2}}{\Delta}+\frac{6 b c}{\Delta^{3}}+\frac{b^{2}}{\Delta^{5}}\right)\left(c \cos ^{2} \varphi-a\right) \operatorname{sen}^{2} \varphi \cos ^{2} \varphi \partial \varphi, \\
& \boldsymbol{S}_{5}=\frac{m}{b c a^{2}} \int_{0}^{\mu}\left(\frac{c}{\Delta^{3}}+\frac{b}{\Delta^{5}}\right)\left(c \cos ^{2} \varphi-a\right)^{2} \cos ^{2} \varphi \partial \varphi .
\end{aligned}
$$

Adottando le notazioni di Legendre per le funzioni ellitliche di prima, e seconda specie, vale a dire

$$
\boldsymbol{F}(\boldsymbol{k}, \varphi)=\int \frac{\partial \varphi}{\Delta}, \quad \boldsymbol{E}(\boldsymbol{k}, \varphi)=\int \partial \varphi \Delta,
$$

gli integrali $\boldsymbol{S}_{0}, \boldsymbol{S}_{1}, \ldots$ sono tutti riducibili ai due trascendenti $\boldsymbol{F}(\boldsymbol{k}, \varphi)$, $\boldsymbol{E}(\boldsymbol{k}, \varphi)$, d'onde ne segue che sostituiti nel valore di $\boldsymbol{S}$, resta pienamente dimostrato che la quadratura della nuova superficie dipende da soli trascendenti ellittici di prima, e seconda specie. Per non allungare di troppo questa addizione, termineremo coll' avvertire, che quantunque ci sia luogo a sperare che i coefficienti finali dei trascendenti ellittici $\boldsymbol{F}(\boldsymbol{k}, \mu), \boldsymbol{E}(\boldsymbol{k}, \mu)$ dopo tutte le riduzioni nel valore di $\boldsymbol{S}$, prendano un' aspetto semplice, contuttociò il risultato si trova nascosto dietro lunghe operazioni analitiche, e delle quali probabilmente ne parleremo in altra circostanza.

Roma 15. Aprile 1846. 